\title{
Strategy implementation through hierarchical couplings in a management control package: an explorative case study
}

\author{
Berend Van der Kolk ${ }^{1}$. Tom Schokker ${ }^{1}$
}

Published online: 23 October 2015

(C) The Author(s) 2015. This article is published with open access at Springerlink.com

\begin{abstract}
We examine how couplings of management control (MC) elements help to implement an organization's strategy. Despite prior research stating that couplings between MC elements form a fruitful soil for further research, empirical studies in this area are still scarce. We draw on coupling theory to explore three hierarchical relations between MC elements, and examine how these couplings help to implement the organization's strategy. We conducted a single case study in a medium-sized Dutch municipality, using interviews, observations and desk research. We identified three different types of couplings which yielded different effects, but were all distinctively helpful in implementing particular parts of the organization's strategy. This study contributes to the MC literature by demonstrating how coupling theory can be operationalized to examine relations between MC elements in an MC package, and by showing how various hierarchical couplings can help to implement an organization's strategy.
\end{abstract}

Keywords Package $\cdot$ Coupling $\cdot$ Hierarchy $\cdot$ Municipality $\cdot$ Public sector $\cdot$ Strategy

JEL Classification M190 $\cdot$ M400 $\cdot$ M410 $\cdot$ M480 $\cdot$ H830

\section{Introduction}

Despite calls to investigate the relations between different management control (MC) elements in organizations, empirical studies in this area are still scarce (Malmi 2013). Prior literature suggested that researching MC elements 'in isolation' gives rise to a

Berend Van der Kolk b.van.der.kolk@rug.nl

1 Faculty of Economics and Business, University of Groningen, Nettelbosje 2, 9747 AE Groningen, The Netherlands 
'fragmented' picture of MC, which may eventually lead to a partial or even wrong understanding of MC elements' effectiveness (Chenhall 2003; see also Bedford and Malmi 2015). We study relations between MC elements at different hierarchical levels to demonstrate the relevance of approaching MC elements 'as a package' (cf. Malmi and Brown 2008). We particularly focus on relations between MC elements that help to realize an organization's strategy by conducting an in-depth case study in a mediumsized Dutch municipality, using thirteen semi-structured interviews, desk research and field observations.

An MC element is used by management to direct employee behavior in such a way that the organization's strategy is realized and its goals are attained (Malmi and Brown 2008). Examples of MC elements are rules and targets for individuals or teams in the organization, but an organization's endeavors to select and train new employees can also be considered MC elements (see e.g. Merchant and Van der Stede 2007). The functioning of individual MC elements, such as the balanced scorecard (Kaplan and Norton 2007, 2005), has been studied often in the prior literature and has provided us with many useful insights. How interrelations between different MC elements help to realize an organization's strategy, however, is an under researched topic in the accounting literature (Marginson 2002). A strong relation between MC elements employed in higher hierarchical levels - which are often more directly linked to the organization's strategy — and those MC elements employed at lower hierarchical levels — which are often more 'concrete'-may enhance the realization of the organization's strategy, whereas a weaker relation between such elements may evoke different, perhaps even negative, effects. So, different relations between MC elements may lead to different effects, which implies a need to study MC elements 'as a package'.

In the extant literature, only a limited number of studies have focused on relations between MC elements (for a review of MC literature see Strauss and Zecher 2013). For instance, Marginson (2002) scrutinized the function of MC elements in a strategy process in a for-profit firm, and focused on the use of different 'categories' of MC elements. More recently, Friis et al. (2015) studied different types of relations between incentive systems and other MC elements in a manufacturing firm. Prior research suggested that coupling theory could be useful in studying relations between different organizational phenomena (Beekun and Glick 2001; Orton and Weick 1990; Abernethy and Chua 1996), however, papers that adopt coupling theory to study MC elements are rare. An exception is a study by van Hengel et al. (2014), who analyzed the coupling of results controls between the political level and the bureaucratic level in 12 Dutch municipalities. They identified 'organizational' and 'institutional' antecedents of the couplings of vertical MC elements and suggested that the couplings between the vertical, i.e. hierarchical, MC elements should be scrutinized in more detail, for instance in a single case study, to enhance our understanding of their relations.

We complement the literature by investigating how MC elements at different hierarchical levels of a public sector organization are coupled to implement strategy. In doing so, we aim to enhance the understanding of the effectiveness of different couplings of MC elements, and thereby benefit both practitioners and academics. Practitioners may particularly find useful our discussions of the different MC elements, their couplings and their effects, and academics may specifically benefit from our operationalization of coupling theory and the insights we provide into the relations between the different 
MC elements used to implement strategy. We conducted an in-depth case study in a medium-sized Dutch municipality, and we specifically paid attention to the implementation of the strategy in day-to-day practices by focusing on one department. In our study we employed thirteen semi-structured interviews, desk research and observations. We identified three different types of couplings that yielded different effects, but were all distinctively helpful for implementing particular parts of the organization's strategy.

In the following section, we introduce coupling theory and build the theoretical framework that we will use to assess the couplings between the various MC elements at the different organizational levels. Subsequently, we convey details about our qualitative research method. In the sections that follow, we introduce our case organization and discuss three hierarchically coupled MC elements. In the final section we provide a discussion, conclusions and suggestions for further research.

\section{Literature overview}

\subsection{Management control and strategy}

Defining $\mathrm{MC}$ is a challenge, since many conceptions of it can be found in the extant literature (Abernethy and Chua 1996; Chenhall 2003; Merchant and Van der Stede 2007). Malmi and Brown (2008, p. 295) discussed various definitions and their particularities in detail, and ultimately embraced a broad notion of MC by stating: "control is about managers ensuring that the behavior of employees [...] is consistent with the organization's objectives and strategy". In this paper, we will use this definition of MC because it clearly depicts the relation that MC elements have with strategy.

In the 1980s, David Otley posed the idea that individual control instruments should be seen as a part of a wider organization control package and that control instruments do not operate in isolation (Otley 1980). In the decades that followed, several authors supported the idea of control instruments 'as a package', and multiple calls for research surveying the linkages between MC elements were made in the literature (Abernethy and Chua 1996; Chenhall 2003; Sandelin 2008; Malmi 2013). Grabner and Moers (2013) discussed the conceptual and empirical issues of researching MC packages, and explicated that MC systems consist of MC elements that are intended to work together, in relation, whereas an MC package is merely the collection of MC elements in a certain entity (e.g. department or organization), and is thus not necessarily intended or designed as such. MC elements can for instance be imposed on employees by managers from different hierarchical levels, who do not necessarily know which other MC elements are used to align the behavior of the subordinates. Recently, van Hengel et al. (2014) distinguished between vertical and horizontal coupling of result controls, and they found that municipalities' control systems were only loosely coupled (vertically) across the various hierarchical levels of the organization, and they recommended a more 'thorough' study of the couplings between MC elements to gain more insight in their interrelations.

MC elements facilitate the alignment of employee behavior with an organization's strategy and objectives. However, the goals of organizations in the private and public 
sectors can be different. Whereas for private sector organizations the ultimate aim can be to maximize shareholder profits, public sector organizations' goals are often more diffuse and they have multiple stakeholders, often with diverging interests. Generally speaking, we could state that public sector organizations want to maximize performance-whatever this 'performance' may be-and minimize costs related to the delivery of their services (Knutsson et al. 2008). To achieve these goals, public sector organizations implement a strategy and use 'packages' of MC elements to make sure that organizational members pursue that strategy effectively (Nutt and Backoff 1995; Malmi and Brown 2008). Several accounting scholars already study strategy. For instance, Skærbæk and Tryggestad (2010) studied strategy and accounting in a government-owned company, and found that accounting instruments were not only instrumental to the implementation of the company's strategy, but also 'shaped' it. More recently, Tucker and Parker (2013) found that the strategy of not-for-profit organizations was mainly implemented through informal, rather than formal, controls. We complement the literature by investigating how MC elements at different hierarchical levels of a public sector organization are coupled to implement the strategy.

To summarize, researching MC elements in the context of their 'interrelations' was recently identified as a gap in the literature (Malmi 2013; Grabner and Moers 2013). It was suggested that we study the relations between the different MC elements using coupling theory (Malmi and Brown 2008; Brown 2005), particularly in the public sector (van Hengel et al. 2014).

\subsection{Coupling theory}

In order to research the linkages between MC elements at different hierarchical levels, we will apply coupling theory (Orton and Weick 1990). Coupling theory can be used as a 'lens' to scrutinize relationships between different organizational elements (Beekun and Glick 2001). These elements can be different organizational phenomena, for instance processes, incentive schemes, personnel management practices and results orientation (Beekun and Glick 2001; Weick 1976). Orton and Weick (1990) identified two variables that determine the type of coupling of two elements: responsiveness and distinctiveness. Responsiveness refers to the extent to which elements respond to each other's changes, whereas distinctiveness refers to the way in which they are different from each other. Orton and Weick (1990) used these notions to categorize different types of couplings, which can be summarized in a two-by-two matrix (see Fig. 1):

"If there is neither responsiveness nor distinctiveness, the system is not really a system, and it can be defined as a noncoupled system. If there is responsiveness without distinctiveness, the system is tightly coupled. If there is distinctiveness without responsiveness, the system is decoupled. If there is both distinctiveness and responsiveness, the system is loosely coupled" (Orton and Weick 1990, p. 205).

Although some of the concepts depicted in the quote, and in Fig. 1, have appealed to researchers, especially the notion of loosely coupled systems, coupling theory has been criticized for its 'cutting-edge mysticism' and it has been claimed that it needs 


\begin{tabular}{|c|c|c|c|}
\hline & & \multicolumn{2}{|c|}{ Responsiveness } \\
\hline & & High & Low \\
\hline \multirow{2}{*}{ 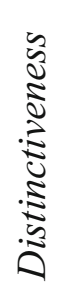 } & $\underset{0}{i \infty}$ & Loosely coupled & Decoupled \\
\hline & ढे & Tightly coupled & Noncoupled \\
\hline
\end{tabular}

Fig. 1 Different types of couplings, based on Orton and Weick (1990)

a more formal approach (Beekun and Glick 2001; Orton and Weick 1990). Beekun and Glick (2001, p. 228) therefore 'formalized' many concepts from coupling theory and analytically informed the theory's concepts, aiming to take it 'beyond a literary metaphor'. Subsequently, Brown (2005) built further on some of the notions from Beekun and Glick (2001) to develop and operationalize 'distinctiveness' and 'responsiveness', key terms in coupling theory. He identified three sub-dimensions for each of the two notions, in order to be better able to specify the different types of couplings, which we will use to operationalize our theoretical model. We will discuss these sub dimensions in more detail in the sections below.

\subsubsection{Distinctiveness}

According to Brown (2005, p. 59), the three sub dimensions that underlie the notion of 'distinctiveness' are focus, use and components. We will now discuss these three sub dimensions now in some detail. First, with the focus of an MC element, Brown (2005) refers to the aims of it, or in other words, the control problem that the MC element addresses. The MC element can, for instance, be aimed at constraining certain selfinterested behavior of the employee, but it can also be aimed at achieving a specific organizational goal. Brown (2005, p. 56) described five specific categories of 'possible focuses', i.e. planning, cybernetic, administrative, socio-ideological, and reward and compensation. However, in this study, we will evaluate in each situation the overlap between the focus of the MC elements, without specifying up front which focuses might exist. This gives us with more freedom to interpret and understand the specific functions of MC elements in particular situations. In our study, focus can, for instance, be low if the two elements have very different focuses, and high if the focuses of the two elements are largely the same. So, (non-)overlapping focuses will yield lower (higher) degrees of distinctiveness.

The second sub dimension is use. Differences in use relate primarily to the timing of the elements. The distinction between ex ante and ex post is paramount for this sub dimension. Organizational rules and procedures are for example MC elements that 
can be used for ex ante purposes, i.e. they can specify desirable behavior 'up front'. Contrastingly, performance evaluations are often used to evaluate past performance and are thus used for ex post purposes. A third possibility is that some elements are used both ex ante and ex post, for instance, budgets that are used both to specify desired results and to evaluate the realized results. When two MC elements are used in the same way, i.e. they overlap in the aforementioned three categories, this lowers their respective distinctiveness.

The notion of components refers to the information the MC elements are built upon (Brown 2005). When two MC elements contain, for instance, individual productivity measures, but are used in different ways, their 'building blocks' are similar, even though their focus may be different. Whenever the components of two elements are the same, we speak of a relatively lower distinctiveness of those elements. When the elements contain different sources of information, the distinctiveness of the two elements is relatively higher (Brown 2005).

According to Brown (2005), MC elements can have high, medium and low levels of distinctiveness when respectively three, two or one of the sub dimensions discussed above are different from each other.

\subsubsection{Dimensions of responsiveness}

The three sub dimensions that together form 'responsiveness' are dependence, directness and strength (Brown 2005). We will discuss these three sub dimensions in this section. First, the dependence of one element on another reflects the relative magnitude of exchange between them, or in other words, how $\mathrm{A}$ is dependent on $\mathrm{B}$, and vice versa (Beekun and Glick 2001). For instance, it can be the case that one MC elements feeds information into another (hierarchically lower) MC element. This enhances the dependence of $\mathrm{A}$ and $\mathrm{B}$.

The second sub dimension of responsiveness is directness, which refers to the 'immediacy' of the relationship between the elements. Beekun and Glick (2001, p. 232) give an example of directness, by referring to persons being 'loosely coupled' if they communicate with each other through different levels of hierarchy, instead of more 'directly'. Translated to MC elements, this implies that directness increases if two MC elements relate to each other more directly. For instance, when information from $\mathrm{A}$ is directly used in element $\mathrm{C}$, directness is higher than when B 'mediates' this relationship between elements $\mathrm{A}$ and $\mathrm{C}$.

The strength of the coupling between two elements is mainly determined by their interaction frequency and intensity, and also by the probability that a change in one causes a change in the other. Frequency can be higher when two MC elements are often used simultaneously and intensity refers to the impact that these elements have on one another. The three parts that make up 'strength' do not all simultaneously need to be high in order to speak of a 'strong' relationship. Beekun and Glick (2001), for instance, nuanced this dimension by stating that certain elements can be loosely and tightly coupled at the same time, e.g. when infrequent interactions have an intense character.

The degree of responsiveness can be viewed as a continuum (Brown 2005). When MC elements have a high level of dependence, directness and strength, they can be 


\begin{tabular}{|c|c|c|c|c|c|}
\hline & \multicolumn{2}{|c|}{$\begin{array}{c}\text { Responsiveness: } \\
\text { Levels of dependence, directness and } \\
\text { strength }\end{array}$} \\
\hline & & & & High & Low \\
\hline \multirow{2}{*}{$\begin{array}{l}\ddot{\theta} \\
0 \\
0 \\
0 \\
0 \\
0\end{array}$} & \multirow{2}{*}{ 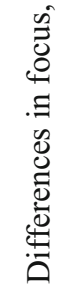 } & \multirow{2}{*}{ 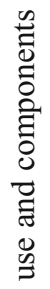 } & 焉 & Loosely coupled & Decoupled \\
\hline & & & 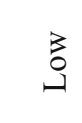 & Tightly coupled & Noncoupled \\
\hline
\end{tabular}

Fig. 2 Different types of coupling including sub dimensions (Orton and Weick 1990; Brown 2005)

considered to be highly responsive to each other. However, it can also be the case that a single sub dimension produces a very high level of responsiveness, for instance, if the strength of the relationship between two elements is very high. Therefore, these sub dimensions should always be used together to scrutinize the responsiveness of two elements. Now that we have this elaborated understanding and operationalization of distinctiveness and responsiveness, we can extend the framework, as shown in Fig. 2. We will use this framework to assess the couplings of MC elements in the empirical parts of this paper. However, first we will discuss the chosen methods for our study.

\section{Methods}

\subsection{Case study research}

We adopted a single case study method to investigate the question: "How do couplings of MC elements at different hierarchical levels facilitate the implementation of an organization's strategy?" When it comes to "why" or "how" questions, conducting a case study is the most appropriate research method (Yin 2014). Malmi and Brown (2008) also referred to this research method to scrutinize the interrelations between $\mathrm{MC}$ elements, since other research types might be less well equipped to study the complexity of the interrelations between different MC elements.

We selected a medium-sized municipality in the Netherlands which we coin 'Easttown', to conduct our explorative case study. We chose this case organization first and foremost because our topic of interest was the coupling of multiple MC elements and how they support the implementation of a strategy, and because employees in municipalities are relatively free-compared to employees in the private sector-to discuss in detail the strategy of the organization with 'outsiders'. This enabled us to obtain rich information in the form of documents that we could analyze and insightful interviews that we were able to conduct. Second, we chose this case because the com- 
bined municipalities in the Netherlands are the second largest public sector employer with almost 180,000 employees. This increases the practical relevance of enhancing insights into MC in this type of organizations. Third, we chose a medium-sized municipality because the trend in the Netherlands is (and has been over recent years) to merge municipalities until they reach a size of at least 100,000 , in order to have 'economies of scale' and sufficient know-how to deal with the recent decentralization of responsibilities from the central government to municipalities.

After our introductory meetings with the municipality's financial controller and a senior policy advisor in the General Policy (GP) department, we were granted access to the organization. We collected data from March 2014 to June 2014. One researcher was present in the case organization during this time period and kept a detailed diary of his activities. We were allowed to attend official meetings (see "Appendices"), and we were given a desk among the other employees of the GP department to work on our research. We did not interfere with work practices of the departmental staff. However, via multiple 'informal' conversations ${ }^{1}$ we could verify information we read in official documents and prepare our interviews.

Thirteen semi-structured interviews were conducted and fully transcribed, the average interview was 50-80 min and the total number of single spaced pages of transcription was 184 . The interviewees were members of staff, lower managers, middle managers and municipal top managers (see Table 1 in "Appendix"), and they were selected because they all were in some way related to the MC relations that we were interested in. ${ }^{2}$

The aim of the interviews was first to reflect on the presence and functioning of the $\mathrm{MC}$ elements that were in place. We then discussed the uses of the different types of $\mathrm{MC}$ elements to ensure we had a grasped which ones were most important for realizing the strategy. We were interested in questions such as: are the MC elements at the department level responsive and distinctive to the strategy formulated at the top-management level? Which control types relate to each other? And how? These questions led us to select three couplings of MC elements that helped to implement strategy at different organizational levels.

\subsection{Data analysis}

We used three sources of data (see Fig. 3), and this allowed us to 'triangulate' the findings of one source with evidence from other sources (Yin 2014). We started analyzing the formal documents obtained from our desk research, and the transcripts of all the interviews. We first structured, summarized and categorized the different types of MC elements that emerged from the collected data. The semi-structured nature of the interviews supported us in constructing this overview. Although each answer was unique, some MC elements were repeatedly mentioned with regard to the realization of the

\footnotetext{
1 For example, during talks in the elevator, near the coffee machine and before and after interviews.

2 We excluded the political level of the municipality from our interviewees, since this was not the focus of the current study. van Hengel et al. (2014) found that the political level has a different understanding of MC than the bureaucracy, which would interfere with the purposes of our study.
} 


\begin{tabular}{lll} 
Interviews & Observations & Desk research \\
\hline 13 semi-structured interviews & $\bullet$ months of day-to-day work & $\bullet$ documents \\
- average length 50-80 minute & observations & $\bullet$ documents related to a.o. \\
$\bullet$ all hierarchical levels of the & $\bullet 3$ 'formal' observations of & strategy, management, past \\
organization included in the & meetings & meetings, all related to both \\
interviews & $\bullet$ numerous 'informal' & the department and the \\
- see also appendix A & observations & $\bullet$ see also appendix C \\
& $\bullet$ see also appendix B & \\
\hline
\end{tabular}

Fig. 3 Overview of the three methods of data collection

municipality's strategy. We applied pattern matching (Miles et al. 2014) to reconstruct the most important types of MC, on the basis of the data and constructed an overview of the MC elements that emerged from our interview data.

In the semi-structured interviews we focused on the (possible) relations between the MC elements and their effects, and we let the respondents reflect on this. Again, using pattern matching, we were able to construct another overview of the couplings between MC elements that emerged from the interviews. This overview was subsequently triangulated with the findings from our direct observations (e.g. informal talks, observations) and formal documents (e.g. organizational budgets and the planning and control cycle), i.e. we conducted data triangulation (Yin 2014, p. 120). More than three couplings came out of this preliminary analysis. However, in order to focus our study and to be able to scrutinize some relations in more detail, we selected the three couplings that contributed most to the strategy implementation of the municipality, according to our interviewees.

We then assessed the three selected couplings using the notions of distinctiveness and responsiveness, as explained in the previous section. In the appendices we give a more elaborate overview of the data that we examined, respectively the interviews (Table 1 in "Appendix"), the observations (Table 2 in "Appendix") and the documents (Table 3 in "Appendix"). We will introduce the case setting in the following section.

\subsection{The case setting}

Easttown is a medium-sized municipality in the north of the Netherlands, which had well over 100,000 inhabitants at the time of our study. When we conducted our research, from January to July 2014, the municipality consisted of one central town (60,000 inhabitants) and thirteen smaller ones, which surrounded it. Easttown wanted to involve its citizens in many aspects of its decision-making processes, as reflected in their core mission statement: "expect more from citizens and be a different government". This statement was widely known by the employees of the municipality, who often referred to it, for example in formal documents and informal conversations. This slogan was illustrative of the way in which the municipality wanted to serve its citizens. By serving the citizens in such a way, the municipality wanted to "create opportunities in cooperation with others". 3

\footnotetext{
3 This is the municipality's mission. This mission is communicated on the website, but also on official documents, such as their annual program budget.
} 
The strategy utilized to carry out this mission is summarized in the core values of the municipality, covered in the acronym "VeRDeR", 4 which can be translated as "moving on". The four key values in this acronym are (translated to English) connecting, (giving) space, decisiveness and (being) realistic. These core values are disseminated through each of the management layers and are regularly communicated to the civil servants. The central ideas in the municipality's mission, vision and strategy documents relate to the idea that society is changing rapidly, and that the municipality should keep pace with these changes. In other words, the starting point should not be what the civil servants want or can do, but what society needs most. This is also connected with the changing role of local governments; from being the 'executor' of tasks to being more and more the 'facilitator' or 'oversight body' of activities (see also ter Bogt et al. 2015). However, having this different role should not mean, according to the municipality's strategy documents, that they should be hesitant in undertaking action, which is why 'decisiveness' was included as a core value of the municipality.

Because the relations between MC elements at different hierarchical levels form the object of our research, we briefly sketch the hierarchical structure of Dutch mediumsized municipalities. van Hengel et al. (2014) distinguished the political level on the one hand and the bureaucracy on the other hand. The political body consists of the municipal council and the 'municipal board', i.e. the mayor and the aldermen. Every four years, the citizens directly choose the municipal council, and the members of the council subsequently choose the alderman. The bureaucracy, which is the focus in this study, consists mainly of three hierarchical levels: top management, middle management and lower management (for further information about management control in Dutch municipalities see van Hengel et al. 2014; van der Kolk et al. 2015).

In the municipal organization of Easttown, approximately 1,200 employees were working at the time of our study, divided over 15 departments. Within the bureaucracy, the top management consisted of the municipal manager, the directors and some staff functions that facilitate management. The middle management consisted of the 15 department heads. The remaining civil servants were led by team managers, which were one level lower in the hierarchy than the department head. Throughout this paper, we consistently use the wordings above to refer to the different managers in the hierarchical levels.

We paid particularly attention to the implementation of strategy through MC elements at the GP department. During the time of our study, no 'major' decisions or changes took place within the municipality or within the department. Therefore, the couplings that we describe in the following sections can be seen as relatively 'stable' representations of the practices related to MC and strategy within the municipality. The GP department consisted of 60 full time equivalent (fte), evenly spread over three teams; economy, social and spatial matters. The employees, who could be regarded as 'policy advisors', were aged between 30 and 64 and their education level was generally high, i.e., most of them held an academic degree. Policy developers were expected to closely follow social developments, both locally and nationally, and to actively con-

\footnotetext{
4 In Dutch: Verbinden, Ruimte, Daadkracht en Realisme. Retrieved from the municipal website on June 2, 2015 .
} 
tribute to the development of policy on a wide range of topics. These developments were complex and dynamic, and could change rapidly.

\section{Three couplings of MC elements}

As indicated above, to study the couplings of MC elements at different hierarchical levels in the organization, we analyzed three couplings of MC elements. These three couplings emerged during our case study, as they proved to be of paramount importance for the implementation of the municipality's strategy, albeit in different hierarchical levels. This could be seen in our observations and in our assessment of the municipality's formal documents, and later also verified during our interviews. We will discuss respectively the couplings between (A) the annual program budget and the individual work plans, (B) the so-called A3 municipal plan and the A3 department plan, and $(C)$ the municipal personnel plan and the conversation cycle. These 'pairs' of MC elements each show different aspects of the organization's strategy: coupling A highlights the connection between higher level program budgets and how they are cascaded down to individual work plans, coupling B is about two MC elements that address many aspects of the strategy at the same time. The form of these MC elements is similar, but they are employed at different organizational levels, with different levels of detail. Coupling $\mathrm{C}$ connects the higher level plans concerning the tasks and performance of personnel with the conversation cycle that is employed at the individual level.

Each of the three pairs of MC elements connect two hierarchical levels. As already mentioned, we are drawing on a broad definition of MC elements, i.e. "control is about managers ensuring that the behavior of employees [...] is consistent with the organization's objectives and strategy" (Malmi and Brown 2008, p. 295). This broad definition incorporates MC elements at 'higher' and 'lower' organizational levels, and particularly focuses on how the behavior of employees is aligned with the organization's objectives and strategy.

The way in which we will discuss each of these three couplings of strategy and MC elements is as follows: first, we describe the two MC elements of interest. Second, we assess their distinctiveness and responsiveness using Brown (2005) sub dimensions and third, we reflect on the type of coupling between the different types of MC elements, how this coupling helped to implement the municipality's strategy, and if it evoked any side effects.

\subsection{Coupling A: the annual program budget and the individual work plans}

The annual program budget and the individual work plan are MC elements that are employed at respectively the organizational level, i.e. municipality, and the individual level. The relation between the two MC elements can best be sketched by a quote from interviewee \#13, who depicts how the municipal strategy is carried out through different hierarchical levels, using different types of MC elements. 
"It [i.e., the strategy] starts with the annual budget program: all programs that aim to realize the municipal goals are listed there. What we want to achieve and how we want to achieve it. The annual budget program is divided in 'products', which are in turn allocated over the fifteen departments. The departments are then responsible for delivering the products that are listed in the annual program budget. So, employees need to work on those, and include them in their personal work plan. In that way, we're able to monitor our performance and exercise control."

The annual program budget is the municipality's plan for the coming year, and it is mainly derived from the four-year executive plan that is written by the municipality's aldermen. The executive plan consists of agreements between the parties that deliver the municipality's alderman (elected every four years), and tends to be more 'abstract' than the annual program budget, which is usually a more practical translation of about one quarter of the executive plan. The 15 departments provide input for their part of the annual program budget. However, the political body has to approve the document and can ask for alterations. After the political body approves it, the annual program budget provides explicit guidance for the municipality's activities as a whole. To indicate the size (and level of detail), in the last few years the annual program budget has consisted of 268 (2012), 252 (2013) and 213 (2014) pages.

The individual work plans are documents that specify the expectations of individual employees for a certain year. These plans are generally in a standard format and they are filled out by the employees. However, this should always be done in consultation with their team leaders. After a conversation with their team leader, employees sometimes have to adapt parts of their original plans before they can be approved. Often, the hours allocated to specific parts of the annual program budget are included in these individual work plans. The relevant subjects in the annual program budget are directly translated and distributed to the individual work plans of the GP staff, who are given a specific number of hours on an annual basis to spend on certain policy programs. Besides regulating the allocation of hours per employee, the individual work plans also pay attention to the 'work style'. It, for instance, includes suggestions about the way in which employees should deal with their day-to-day activities, by repeating and applying the municipality's core values and identity. Interviewee \#1 comments:

"First there's the department plan, which is highly connected to the annual program budget [one level higher]. One level lower than the department plan, there's the team plan, and one level lower you'll find the individual work plans. Those are created on a yearly basis. That's the place where I write up how many hours I'm planning to work on each of my activities. That's my guideline for the year."

Individual work plans usually consist of ten categories, which contain information about the link between the municipality's strategy and the individual's tasks, i.e., employees are specifically asked by their supervisors to explicate the link between the strategy and their individual plans for a given time period. The plan is usually between three and eight pages long, and it always ends with information about the individual's planned hours for a given period. 


\subsubsection{Distinctiveness coupling A}

The three sub dimensions that together form distinctiveness are use, focus and components. Firstly, the use of both MC elements is 'ex ante', that is to say, both elements aim to prescribe actions before they actually take place. However, both MC elements are also used to evaluate a period in the past, but because this ex post use is the same for both elements, their 'use' remains similar. The annual program budget is used to plan the municipality's activities for a given year, while the individual work plans are used to plan the activities of the individual employees. This indicates that the focus of the two MC elements is different: the former is aimed at a higher organizational level and the latter is focused on the individual. The focus of the two elements is thus different. The components of the annual program budget and the individual work plans are different: the annual program budget consists of many different programs that contain actions that the municipality should take in the next year, whereas the individual work plans consist only of the planned activities of the individual employees. Although the 'building blocks' of the individual work plans are partly the same as those of the annual program budget, since both are documents that 'plan' activities up front, the MC elements used to direct employee behavior are different than the ones that guide the organization's strategy at the higher organizational level.

Following our theoretical framework, our impression is that the distinctiveness of the two elements can be considered as 'medium to high', since two out of the three sub dimensions show results that point at high levels of distinctiveness.

\subsubsection{Responsiveness coupling A}

All interviewees recognized the influence of the strategy, as set out by the municipality's top management and the political body, on their daily activities. The annual program budget bears many of the top management's ideas, and serves as a vehicle to translate these ideas into operational work plans. The following quote from interviewee \#7 sketches the responsiveness of the individual work plans and the annual program budget.

"The annual program budget clearly indicates what needs to be done in a given year. For instance, program two, which is about 'Economy', discusses the actions and activities that need to be done, what it is we have to work on. These things need to be ready at the end of the year, and the corresponding actions and activities are subsequently translated into the individual work plans."

The three sub dimensions that together form responsiveness are dependence, directness and strength. The dependence of individual work plans on the budgeting program is experienced as relatively high. For example, the annual program budget states that more jobs should be created for citizens over 55 years. Therefore, one of the policy advisors within the GP department has to include this task in his or her individual work plan, and design (and perhaps implement) actions that contribute to the achievement of this goal. 
Although the annual program budget serves as an input for the individual work plans, this relation is rather indirect, as indicated by interviewee \#8, who is a team leader.

"Through the hierarchical levels, the organizational goals are being communicated. We first look at the goals in the annual budget program, and translate them in the departmental program. Subsequently, use these in the team and individual work plans."

In other words, although the relation is 'mediated' by other MC elements at different hierarchical levels, the annual budget program still affects the individual work plan in that it prioritizes actions in terms of subjects and courses of action. We, therefore, have the impression that the directness between the two MC elements should be viewed as 'moderate'. The strength of the relation between the two management controls also seems to be high. The strength of the coupling is determined by the frequency, intensity and the probability that change in one element will cause change in the other. The frequency of the interaction between the elements is about one or two times per year, however, because they are also only 'designed' once every year, we consider this to be a relatively high frequency. The intensity of this relation can be considered high as well, because the annual work plan is an important input for the individual work plans. Since the annual program budget is the input of the individual work plans, the probability of change is high because work plans are adjusted to the budget program.

Given the scores of this coupling on the three dimensions (high, moderate and high), we consider the responsiveness of this coupling to be high.

\subsubsection{General impression and appreciation of coupling A}

Following our categorization, the annual program budget and the individual work plans are relatively distinctive and have a high level of responsiveness, therefore we classify them as 'loosely coupled'. The relation between the two MC elements is appreciated, i.e. positively valued, by many of the interviewees, although it is sometimes also acknowledged that the process of connecting the individual work plan with the team, department and municipal plans is too time-consuming. The employees involved in this process are sometimes a bit 'tired' of it, because too many meetings are sometimes planned to discuss these matters. Interviewee \#8, a team leader, comments:

"I really like the connection between the annual program budget, the department plan, the team plan and the individual work plan. [...] It's just a very nice way of working. Of course, it's not always consistent, but all in all I think it is working quite well. However, some people also mock on it, mainly because we are sometimes too busy to discuss our plans together. But when I then speak to these people in private, they are all very enthusiastic, because we pay particular attention to their individual situations." 


\subsection{Coupling B: the $\mathrm{A} 3$ municipal plan and the $\mathrm{A} 3$ departmental plan}

The 'A3 municipal plan' and the 'A3 departmental plan' are both based on 'EFQM excellence models', 5 and fit on a single A3 sheet of paper (see Fig. 5 in "Appendix" for the anonimized A3 municipal plan). An EFQM excellence model consists of nine categories that are designed in such a way that they help to depict the crucial elements of the municipality's strategy, actions and goals. Five categories are framed as 'enablers', such as leadership, strategy and people, the remaining four categories of the model consist of results, like customer, society and business results.

The A3 municipal plan communicates the expectations of top management for other parts of the organization. It is designed by the municipality's top-management and it is an instrument that communicates and bears the strategy of the municipality. However, unlike the annual budget program, this A3 municipal plan is designed and used by the municipality's bureaucracy and does not need the approval of the political level. The A3 municipal plan consists of three main parts. The first contains the mission, vision, the critical success factors of the organization, and the cultural core values. The second section translates the mission, vision and success factors into the requirements of leadership, strategy and policy, and the management of resources, employees and processes. The third and last section includes goals about employees, client satisfaction, society, and the council and aldermen. These goals reflect the ambitions of the organization and are evaluated at the end of every calendar year. The goals are, however, very general: i.e., they are written down in such a way that every department can recognize itself and has sufficient freedom to translate them to their own specific situation. After a calendar year the A3 municipal plan is revised at the organizational level, which then results in changes in the A3 plans in the different hierarchical levels.

The $A 3$ departmental plan is designed by the department manager, team managers and departmental staff. ${ }^{6}$ The aforementioned three parts are also visible in the A3 departmental plan. However, they are now more specific and designed so as to fit the tasks and characteristics of the team. Because staff is also involved in translating the A3 municipal plan into the A3 departmental plan, they are generally very familiar with it. Interviewee \#5 comments on the A3 plans and the other plans that are used to exercise MC:

"There's an A3 plan of the department, we hung it near the coffee table. The plan describes what we want to achieve as a department. We also have a team plan and an individual work plan, but they are more 'tailor made': you can see those individual plans as a personal 'shopping list': those are the things you should be doing."

\footnotetext{
5 EFQM stands for European Foundation for Quality Management. The EFQM excellence model was developed in the eighties and nineties by a variety of business leaders. For more information on the model and its applications we refer to http://www.efqm.org.

6 This varies per department within the municipality. However, we focus on the GP department in our study.
} 


\subsubsection{Distinctiveness coupling $B$}

When comparing the two MC elements in terms of use, focus and components, many similarities can be drawn. The use of the two A3 plans is similar, in that they both have_-primarily — an 'ex ante' function and direct the municipality's and department's actions. However, both A3 schemes are also, to a small extent, used as 'evaluative' (ex post) instruments to assess to what extent the plans for a given year are realized. The feedback on both A3 plans is used in order to develop the A3 plans for the next year. All in all, their use is relatively similar. The focus is a bit different, since the scope of the municipal plan is wider than the organizational plan, which is far more specific. However, since the two MC elements do not focus on completely different areas (they overlap to a large extent, although the A3 departmental plan is more specific) their focus can still be seen as relatively similar. The components of the two A3 plans are completely similar, since they both use the same format.

To summarize, differences in focus, use and components are relatively low, which indicates a low level of distinctiveness.

\subsubsection{Responsiveness coupling $B$}

The goals and ambitions in the A3 municipal plan function as the basis for the A3 department plan. Some goals are even literally copy-pasted. For instance, both A3 plans mentioned "in the end of 2014, the planned budget cuts are $100 \%$ realized". Other goals are translated into the specific context of the GP department. For example, the municipality's ambition states: "We want to be a municipal organization that is visibly involved with the society". At the department level, this ambition is operationalized and translated into: "We want to offer internships, offer places for work experience, have a minimum of six inspirational lectures that are better attended than last year." Because of these types of translations, the A3 department plan is highly dependent on the A3 municipal plan, and the strength of this relation is also considered high. Directness can also be considered high, since there are no $\mathrm{MC}$ elements that mediate between these two A3 plans.

All in all, the level of responsiveness between the A3 municipal plan and the A3 department plan is high, indicated by high levels of dependence, strength and directness.

\subsubsection{General impression and appreciation of coupling $B$}

The A3 municipal plan and the A3 department plan are highly responsive to each other, and show a low distinctiveness. Following our theoretical framework, these two MC elements are 'tightly coupled'.

The more general ideas created by the municipal top management are dispersed over the 15 departments using the A3 plans, which are subsequently also used by teams and individuals. These translations are necessary to operationalize and implement the municipality's strategy. Many interviewees recognized the positive effects of the coupling of the A3 plans throughout the organization, as clearly indicated by interviewee \#10, the department head of GP: 
"I've seen many planning and control systems in my life, but the A3 plans are just great. You can easily hang them somewhere. Here it is for instance displayed in the departmental kitchen. Of course...it also depends on how it's used, but I think we can be pretty proud at it. It's a beautiful system which connects many hierarchical levels with each other."

He further illustrated why the A3 plans help in implementing strategy:

"The A3 model is a model that we used the last ten years in order to continue speaking the same language in the organization. It is also a model that emphasizes all elements of leadership, process and employees. The EFQM model is the basis for the A3 model. Thus, in that way cohesiveness is emerging."

Using these tightly coupled MC elements creates a sense of cohesiveness within the municipality. The form of the A3 plans helps employees to recognize the municipality's strategy in their own department and team, and hence, enhances their 'identification' with the municipality.

\subsection{Coupling C: municipal personnel plan and conversation cycle}

Both the municipal personnel plan and the conversation cycle are MC elements that deal with the 'human resources' of the municipality. However, the former is a general and broad plan that contains the overall direction, whereas the latter is a specific MC element that is employed at the individual level. The municipal personnel plan was developed by the municipality's top management, together with its staff. The aim of the plan was to manage its 'human resources' in the long term. It contains information on topics such as the size, quality and composition of the workforce, but it also includes expectations regarding employees and it expresses some of the municipality's core cultural values.

The conversation cycle refers to subordinates having three conversations with their superior per year. In these conversations, respectively expectations, progress and evaluation are the topics addressed. Interviewee \#8 further elaborates:

"As stated in the new municipal personnel plan, we are implementing conversation cycles. Each employee speaks with the team manager at the beginning of the year about a personal development and work plan. During the year, there's a second meeting that addresses questions such as "Where are you now? Do you need anything? Do we need to adjust the plan, or do you need some help of a colleague?" At the end of the year, the products they 'made' will be evaluated. They'll have to discuss which things they didn't achieve and why. You could call it a sort of performance appraisal."

We will now scrutinize the relation between these two MC elements, following the same structure as in the previous couplings. 


\subsubsection{Distinctiveness of coupling $C$}

Distinctiveness consists of the sub dimensions use, focus and components. Regarding use, the municipal personnel plan is considered an 'ex ante' MC element that prescribes which actions should be taken over the longer term, whereas the conversation cycle is used both 'ex ante' and 'ex post'. This is because these conversations function both as guidance for future behavior, as well as an evaluation of past behavior, as illustrated by interviewee $\# 2$ :

"The conversations with my team leader are very positive. They function as a kind of 'direction' to me; he expects me to conduct some tasks, and sometimes I succeed in that and sometimes not. And of course, I expect him to provide me with feedback. So, yes, I really appreciate these conversations."

Therefore, the use of these MC elements is mostly different. The focus of the two elements is also different, since the municipal personnel plan provides a general image for the whole organization, whereas the conversation cycle, as used in the GP department, is highly specific and differentiated per employee. The components of the two MC elements differ as well. Whereas the municipal personnel plan is an informative policy document that contains rules, routines and protocols, the conversation cycle is a dynamic dialogue between two organizational members, taking place three times a year.

Therefore, the overall distinctiveness between the two MC elements is considered high.

\subsubsection{Responsiveness of coupling C}

The conversation cycle was partly based on ideas from the municipal personnel plan, which may indicate some responsiveness of the two elements. However, when reviewing the sub dimensions dependence,directness and strength, we found that for conversation cycles the team managers often only superficially use the prescribed formats from the municipal personnel plan. Because team leaders have a lot of autonomy in the way they have conversations with their subordinates, the level of dependence is considered to be low to moderate. Because the conversations had to be reported in a semi-structured form, ideas from the municipal personnel plan did fed directly into parts of the reports in the conversation cycle. As this may have affected the conversation cycles (but not necessarily) directness was considered low to moderate. The strength of the relations was rather low, since the format of the conversation cycle did not very change much even though the team leaders, because of their autonomy, have the ability to deviate from the formats provided in the official municipal personnel plan. The likelihood that a change in the municipal personnel plan would affect the individual conversation cycles was moderate, and the intensity of this relation was considered low.

All in all, the level of responsiveness of this coupling can be regarded as 'low' to 'moderate'. 


\subsubsection{General impression and appreciation of coupling $C$}

As distinctiveness is considered high and the responsiveness is low to moderate, the municipal personnel plan and the conversation cycles can be categorized as 'decoupled'. This decoupled relation was experienced very differently within the organization. Some interviewees, for instance, valued the conversation cycle because it enabled a discussion about their progress during the year. They indicated that these three meetings were a very important mechanism in order to keep track of the expectations of management, to give attention to the progress made regarding these expectations and to discuss personal or career development. All interviewees mentioned this cycle immediately when we asked general questions about MC, and, within the GP department, the conversation cycle and its links to the municipal personnel plan were appreciated. However, as indicated by the following quote from interviewee \#8, the prescribed format of the conversations was often neglected:

"We have to use very strict formats. I've got to admit that I don't always stick to those. There are subjects on them that are not relevant for my team members at all. In those situations it's more like: "What shall we fill in here?" and then it's okay. However, there are also other subjects that are discussed in more detail, and those we put into other boxes. In that sense, we don't always stick to the strict formats."

Interviewees often mentioned that the content of the conversation cycle was highly very specific to each person, and that managers in the GP department did not adopt a 'one-size-fits-all' approach. Although this practice of 'decoupling' the two MC elements was evaluated positively by the interviewees, it also increased the 'distance' and 'alienation' towards the staff departments, which designed the municipal personnel plan. The supervisors only paid 'lip service' to some aspects of the reports in the conversation cycle, while relying on their own norms, values and priorities to have good conversations with their subordinates.

In Fig. 4, we depict the impressions we have of the three different couplings of different MC elements: first we categorized the couplings on the basis of our impressions of the dimensions of interest, and second, we stated some of the striking findings regarding the interviewees' impression of the couplings, and in particular whether these impressions are positive.

\section{Discussion and conclusions}

In this section, we discuss and compare the three different hierarchical couplings from the findings section and connect our findings to the extant literature.

In all relations studied, we observed couplings that helped to 'cascade' the organization's strategy to lower hierarchical levels, irrespective of the type of coupling (loosely coupled, tightly coupled or even decoupled, see Fig. 3). Through all these couplings, the sometimes 'abstract' formulations of strategy were operationalized for and by the organizational members. Clearly linking different $\mathrm{MC}$ elements with each other throughout an organization can increase the identification of employees with the organization's goals and strategy, as reflected in the interviewees' responses. Iden- 


\begin{tabular}{|c|c|c|c|}
\hline Coupling & $\mathbf{A}$ & B & C \\
\hline Higher level MC element & Annual program budget & A3 municipal plan & Municipal personnel plan \\
\hline Lower level MC element & Individual work plan & A3 department plan & Conversation cycle \\
\hline Categorization & Loosely coupled & Tightly coupled & Decoupled \\
\hline Distinctiveness & Medium/High & Low & High \\
\hline - Use & Similar & Similar & Different \\
\hline - Focus & Different & Mostly similar & Different \\
\hline - Components & Different & Similar & Different \\
\hline Responsiveness & High & High & Low/Moderate \\
\hline - Dependence & High & High & Low/Moderate \\
\hline - Directness & Moderate & High & Low/Moderate \\
\hline - Strength & High & High & Low \\
\hline $\begin{array}{r}\text { Interviewees' general } \\
\text { impression }\end{array}$ & $\begin{array}{l}\text { Employees enthusiastic because } \\
\text { of personal attention and } \\
\text { identification with municipal goals } \\
\text { - Sometimes relating the two is } \\
\text { considered too time-consuming }\end{array}$ & $\begin{array}{l}\text { Employees learn to 'speak the } \\
\text { same language', it increases } \\
\text { cohesiveness in the organization } \\
\text { - The A3 plans connect } \\
\text { hierarchical levels and increase } \\
\text { identification }\end{array}$ & $\begin{array}{l}\text { - 'Lip service' is paid to aspects } \\
\text { of municipal personnel plan } \\
\text { - Too strict format increases } \\
\text { distance between employees and } \\
\text { top management }\end{array}$ \\
\hline
\end{tabular}

Fig. 4 Overview of findings

tification with the organizational goals may foster so-called 'steward-like' behavior, i.e. behavior that is aimed at serving the organization (cf. Hernandez 2012). Also, the joint development and translation of strategy to other levels in the organization had positive effects, which is consistent with the extant literature (Groen et al. 2012). This finding suggests that communicating and co-developing can be useful instruments to increase the identification of employees, and thus to 'reinforce' the effectiveness of both MC elements. However, we also found that organizational members may at some point become 'tired' of the many conversations and 'co-developments' that are needed to translate or cascade the strategy throughout the hierarchical levels, as explicitly mentioned regarding coupling A. Thus, we propose that this positive relation only holds until a point of 'saturation' is reached: after this point communication and co-development may start to have counterproductive effects.

Some couplings of MC elements seem more suitable to cascade organizational strategy down the hierarchy than others, as indicated by the described side effects of these couplings. For instance, while a 'tight' coupling between the A3 plans (coupling B) enhanced the organizational cohesiveness, the decoupled relation between the municipal personnel plan and the conversation cycles (coupling $\mathrm{C}$ ) seemed to have the opposite effect, i.e. employees felt that the formats used were sometimes too strict. A possible explanation may be that the former was a coupling between two MC elements 'higher in the hierarchy', whereas the latter dealt with individual employees, who perhaps feel more 'unique' than departments and therefore dislike standardized formats.

When comparing our findings with the extant literature, we see that there are a few striking findings. Some hierarchically coupled MC elements are for instance 'tightly' coupled, while van Hengel et al. (2014) only found 'loose' relations between vertically coupled MC elements within municipalities. A possible explanation for this is that van Hengel et al. (2014) studied the perceptions of political bodies in their analysis, while we focused on the 'bureaucracy' of the municipality — although the MC elements studied were all somewhat related to the politically informed and approved program budget.

We categorized coupling C as 'decoupled', and we observed that team managers used their autonomy to infuse the content of the conversation with their own inter- 
pretations of relevance and importance. In other words, it seemed that it was only the structure of three yearly conversations that was cascaded down in the organization, while many supervisors and subordinates filled in the content differently. This shaping of the conversations to the specifics of the situation-which was 'taken' by supervisors and their subordinates rather than 'granted' to them-was greatly valued by employees. This reflects the perception that it is sometimes necessary to deviate from the standardized formats prescribed by other parts of the organization. This finding is consistent with ter Bogt et al. (2015), who also criticized MC elements of a 'uniform' nature, which do not allow for differentiation, because specific situations can require different types of MC.

Finally, we also want to evaluate the use of coupling theory to assess relations between MC elements in an organization. Firstly, we can state that Brown (2005) operationalization of the sub dimensions of distinctiveness and responsiveness helped us to structure our analyses of the different relations, and increased the comparability of the different couplings (A, B and C). Thereby, it allowed our analysis to describe in much detail the couplings between two MC elements. We proposed and employed a slight adaption to Brown (2005) framework, by using relative overlap as a criterion to assess the focus of individual elements. This provided us with more degrees of freedom to examine particular MC elements rather than the five predetermined categories. We suggest a 'continuum', from low to high, which indicates degrees of overlap between different MC elements to indicate the differences in focus.

Secondly, and more generally, we think that coupling theory is a useful theory to assess relations between MC elements, and is consistent with suggestions of Beekun and Glick (2001) and Malmi and Brown (2008). Coupling theory, as employed in this study, has the potential to further inform the distinction between an MC system and an MC package. Grabner and Moers (2013) claim that it is possible to view all $\mathrm{MC}$ elements used by an organization as the MC package and that an MC system is only formed when MC elements "are interdependent and the design choices take these interdependencies into account" (Grabner and Moers 2013, p. 408). If these requirements are fulfilled, the $\mathrm{MC}$ elements are responsive to each other and their relation is either tightly or loosely coupled. It is possible, however, that two MC elements were not designed together, and are still tightly or loosely coupled (and thus interdependent). This is, for instance, the situation when two MC elements are employed at different hierarchical levels at different times, and at some point start to affect one another. Following Grabner and Moers (2013), such a group of interdependent MC elements would not classify as an 'MC system'. Yet, because the MC elements are interdependent it could be argued that they work 'as a system', even though they were not designed or intended as a system. Following this line of argument, we think that it is possible to distinguish an 'intended' MC system, which is designed taking the interdependencies into account, and an 'unintended MC system', which is the result of interdependent MC elements working together 'as a system'.

There are also limitations of using coupling theory. For instance, it does not allow for 'tensions' or 'paradoxes', such as a coupling that simultaneously has low and high responsiveness. Beekun and Glick (2001) identified this as an issue in their study, when they described intense and infrequent communication between two elements, which 
then should be indicated as having both 'low' and 'high' strength. So, this is a limitation to the current state of coupling theory, which needs further attention in future research.

To conclude, we explored couplings of higher-level MC elements and lower-level operational MC elements in the MC package of a municipality, and assessed how they helped to realize strategy. We contributed to the MC literature by showing that hierarchical relations of MC elements can be very different in terms of their type of coupling (loosely coupled, tightly coupled or decoupled), but still can be helpful in implementing the organization's strategy. In the current study, these different couplings produced different effects within the organization. Furthermore, we contributed to the literature that focuses on middle management by showing how $\mathrm{MC}$ elements facilitate the interaction between top and middle management in a public sector setting (cf. Raes et al. 2011; Marginson 2002). In particular, we demonstrated how these interactions help to implement organizational strategy. We believe that our study is one of the first to use coupling theory to assess relations in an MC package and how they help to realize an organization's strategy.

Acknowledgments We would like to thank Hugo van Hengel, Henk ter Bogt, Bob Scapens and the two anonymous reviewers for their constructive comments on earlier versions of this paper. We also thank the municipality of 'Easttown' and its employees for their cooperation during the research process.

Open Access This article is distributed under the terms of the Creative Commons Attribution 4.0 International License (http://creativecommons.org/licenses/by/4.0/), which permits unrestricted use, distribution, and reproduction in any medium, provided you give appropriate credit to the original author(s) and the source, provide a link to the Creative Commons license, and indicate if changes were made.

\section{Appendix}

See Tables 1, 2, 3 and Fig. 5.

Table 1 List of interviews

\begin{tabular}{lllll}
\hline Interview nr. & Function level interviewee & Date & Length (min) & Age category \\
\hline 1 & Account manager & $09 / 04 / 2014$ & 76 & $30-40$ \\
2 & Policy employee 1 & $14 / 04 / 2014$ & 69 & $30-40$ \\
3 & Policy employee 2 & $15 / 04 / 2014$ & 62 & $40-50$ \\
4 & Policy advisor 1 & $10 / 04 / 2014$ & 58 & $40-50$ \\
5 & Policy advisor 2 & $09 / 04 / 2014$ & 82 & $60-65$ \\
6 & Policy advisor 3 & $09 / 04 / 2014$ & 84 & $50-60$ \\
7 & Team manager 1 & $09 / 05 / 2014$ & 53 & $50-60$ \\
8 & Team manager 2 & $22 / 04 / 2014$ & 76 & $40-50$ \\
9 & Team manager 3 & $19 / 04 / 2014$ & 62 & $50-60$ \\
10 & Department head & $25 / 04 / 2014$ & 72 & $50-60$ \\
11 & General advisor to department head & $13 / 05 / 2014$ & 68 & $50-60$ \\
12 & HR advisor to department head & $13 / 05 / 2014$ & 56 & $50-60$ \\
13 & Member top management & $20 / 05 / 2014$ & 57 & $40-50$ \\
\hline
\end{tabular}


Table 2 Observed meetings

\begin{tabular}{|c|c|c|c|}
\hline Date & Name & $\begin{array}{l}\text { Length } \\
\text { (min) }\end{array}$ & Comments \\
\hline $15 / 04 / 2014$ & $\begin{array}{l}\text { Business } \\
\text { management } \\
\text { meeting }\end{array}$ & 120 & $\begin{array}{l}\text { The } 15 \text { department managers and the concern controller attended } \\
\text { this meeting. In these meetings, topics that concern the whole } \\
\text { organization are discussed. In addition, discussions about the } \\
\text { question 'how to motivate employees to participate in attaining } \\
\text { the organization's goals' were facilitated }\end{array}$ \\
\hline $16 / 04 / 2014$ & $\begin{array}{l}\text { Management } \\
\text { team GP } \\
\text { department }\end{array}$ & 160 & $\begin{array}{l}\text { This meeting was attended by all staff members, all team } \\
\text { managers, the business manager and the HR advisor of the GP } \\
\text { department. Actual topics were being discussed and possible } \\
\text { approaches to address current challenges were discussed }\end{array}$ \\
\hline $06 / 05 / 2014$ & $\begin{array}{l}\text { Team meeting } \\
\text { spatial } \\
\text { development }\end{array}$ & 120 & $\begin{array}{l}\text { This meeting was attended by advisors of the team 'spatial } \\
\text { development' and its team manager. Decisions that were made } \\
\text { in the political or MT meetings were communicated here to } \\
\text { team members. Furthermore, expectations of the team were } \\
\text { discussed. Questions such as 'What is the distribution of policy } \\
\text { area's', and 'Is this optimal' were discussed. Subordinates were } \\
\text { actively involved in this process }\end{array}$ \\
\hline
\end{tabular}

Table 3 List of documents assessed

\begin{tabular}{|c|c|}
\hline Name & Subject \\
\hline A3 municipal plan 2014 & $\begin{array}{l}\text { EFQM model that expressed targets and subjects that deserved } \\
\text { attention on the organizational level }\end{array}$ \\
\hline A3 department plan 2014 & $\begin{array}{l}\text { EFQM model-'translated' from the municipal level to the } \\
\text { department level }\end{array}$ \\
\hline A3 team plan 2014 & EFQM model for a specific team \\
\hline Absenteeism monitor & $\begin{array}{l}\text { System that monitors the reporting frequency, amount of days and } \\
\text { percentage of absenteeism }\end{array}$ \\
\hline Benchmark safety 2013 & $\begin{array}{l}\text { Formal document in which municipalities are compared to each } \\
\text { other regarding safety issues }\end{array}$ \\
\hline Executive report & $\begin{array}{l}\text { Report made for the political level that summarizes the most striking } \\
\text { outcomes of the management report }\end{array}$ \\
\hline Executive agreement 2010-2014 & $\begin{array}{l}\text { Agreement between the 'coalition parties', in which—although in } \\
\text { abstract terms - the goals for four years are written down }\end{array}$ \\
\hline Municipal program budget 2014 & $\begin{array}{l}\text { Formal document that describes the planning and goals for } 2014 \text {, } \\
\text { based on the executive agreement }\end{array}$ \\
\hline Credit monitoring & System that monitors the progress on budgets \\
\hline Individual work plans & $\begin{array}{l}\text { Individual work planning with an arc time of one year. Concerning } \\
\text { the input from what has to be done and how it should be done }\end{array}$ \\
\hline Mandate register 2012 & Delegation of decision rights of the organization \\
\hline Management report & $\begin{array}{l}\text { Report of the scores of several crucial indicators, e.g. invoice } \\
\text { monitors, absenteeism monitor, financial budgets }\end{array}$ \\
\hline Organization chart 2012 & Overview of the municipality's structure \\
\hline Collaborate with the society & $\begin{array}{l}\text { Guide for employees of the GP department, containing information } \\
\text { about a method to write policies while involving citizens }\end{array}$ \\
\hline Municipal personnel plan & $\begin{array}{l}\text { Formal document where the current situation and the course of } \\
\text { action concerning human resources is described }\end{array}$ \\
\hline WBH-system & Web-based-hour registration system \\
\hline
\end{tabular}




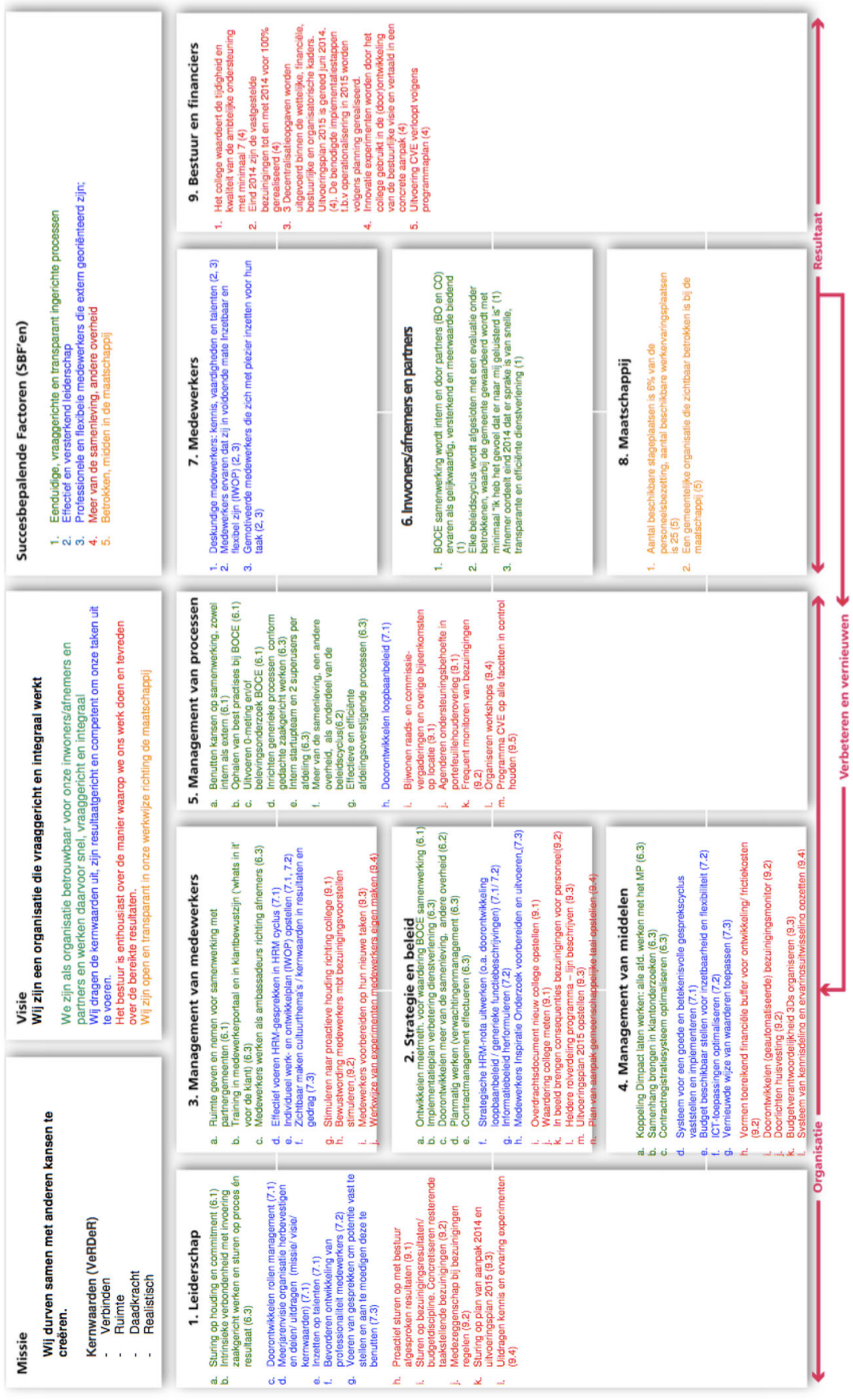

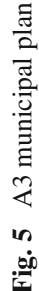




\section{References}

Abernethy, M. A., \& Chua, W. F. (1996). A field study of control system "redesign": The impact of institutional processes on strategic choice. Contemporary Accounting Research, 13, 569-606.

Bedford, D. S., \& Malmi, T. (2015). Configurations of control: An exploratory analysis. Management Accounting Research, 27, 2-26.

Beekun, R. I., \& Glick, W. H. (2001). Organization structure from a loose coupling perspective: A multidimensional approach. Decision Sciences, 32, 227-250.

Brown, D. A. (2005) Management control systems as a coupled package: An analytical framework and empirically grounded implications. $\mathrm{PhD}$ thesis, Sydney: University of Technology.

Chenhall, R. H. (2003). Management control systems design within its organizational context: Findings from contingency-based research and directions for the future. Accounting, Organizations and Society, $28,127-168$.

Friis, I., Hansen, A., \& Vamosi, T. (2015). On the effectiveness of incentive pay: Exploring complementarities and substitution between management control system elements in a manufacturing firm. European Accounting Review, 24, 241-276.

Grabner, I., \& Moers, F. (2013). Management control as a system or a package? Conceptual and empirical issues. Accounting, Organizations and Society, 38, 407-419.

Groen, B. A. C., Wouters, M. J. F., \& Wilderom, C. P. M. (2012). Why do employees take more initiatives to improve their performance after co-developing performance measures? A field study. Management Accounting Research, 23, 120-141.

Hernandez, M. (2012). Toward an understanding of the psychology of stewardship. Academy of Management Review, 37, 172-193.

Kaplan, R. S., \& Norton, D. P. (2007). Using the balanced scorecard as a strategic management system. Harvard Business Review, 85, 150-161.

Kaplan, R. S., \& Norton, D. R. (2005). The balanced scorecard: Measures that drive performance (cover story). Harvard Business Review, 83, 172-180.

Knutsson, H., Mattisson, O., Ramberg, U., \& Tagesson, T. (2008). Do strategy and management matter in municipal organisations? Financial Accountability and Management, 24, 295-319.

Malmi, T. (2013). Management control as a package: The need for international research. Journal of Management Control, 23, 229-231.

Malmi, T., \& Brown, D. A. (2008). Management control systems as a package-opportunities, challenges and research directions. Management Accounting Research, 19, 287-300.

Marginson, D. E. W. (2002). Management control systems and their effects on strategy formation at middlemanagement levels: Evidence from a U.K. organization. Strategic Management Journal, 23, 10191031 .

Merchant, K. A., \& Van der Stede, W. A. (2007). Management Control Systems. Edinburgh Gate, England: Pearson Education Limited.

Miles, M. B., Huberman, A. M., \& Saldaña, J. (2014). Qualitative data analysis: A methods sourcebook. California: Sage Publications Inc.

Nutt, P. C., \& Backoff, R. W. (1995). Strategy for public and third-sector organizations. Journal of Public Administration Research and Theory: J-PART, 5, 189-211.

Orton, J. D., \& Weick, K. E. (1990). Loosely coupled systems: A reconceptualization. Academy of Management Review, 15, 203-223.

Otley, D. T. (1980). The contingency theory of management accounting: Achievement and prognosis. Accounting, Organizations and Society, 5, 413-428.

Raes, A. M. L., Heijltjes, M. G., Glunk, U., \& Roe, R. A. (2011). The interface of the top management team and middle managers: A process model. Academy of Management Review, 36, 102-126.

Sandelin, M. (2008). Operation of management control practices as a package - a case study on control system variety in a growth firm context. Management Accounting Research, 19, 324-343.

Skærbæk, P., \& Tryggestad, K. (2010). The role of accounting devices in performing corporate strategy. Accounting, Organizations and Society, 35, 108-124.

Strauss, E., \& Zecher, C. (2013). Management control systems: A review. Journal of Management Control, 23, 233-268.

ter Bogt, H. J., van Helden, G. J., \& van der Kolk, B. (2015). Challenging the NPM ideas about performance management - selectivity and differentiation in outcome-oriented performance budgeting. Financial Accountability and Management, 31, 287-315. 
Tucker, B. P., \& Parker, L. D. (2013). Out of control? Strategy in the NFP sector: The implications for management control. Accounting Auditing Accountability Journal, 26, 234-266.

van der Kolk, B., ter Bogt, H. J., \& van Veen-Dirks, P. M. G. (2015). Constraining and facilitating management control in times of austerity: Case studies in four municipal departments. Accounting, Auditing and Accountability Journal, 28, 934-965.

van Hengel, H., Budding, T., \& Groot, T. (2014). Loosely coupled results control in dutch municipalities. Financial Accountability and Management, 30, 49-74.

Weick, K. E. (1976). Educational organizations as loosely coupled systems. Administrative Science Quarterly, 21, 1-19.

Yin, R. K. (2014). Case study research. London: Sage Publications Inc. 